\title{
Rational design of new electrolyte materials for electrochemical double layer capacitors
}

\author{
Christoph Schütter ${ }^{a, b}$, Tamara Husch ${ }^{c}$, Venkatasubramanian Viswanathan ${ }^{d}$, Stefano Passerini ${ }^{a, b}$, Andrea \\ Balducci $^{a, b^{*}}$ Martin Korth ${ }^{c^{*}}$ \\ ${ }^{a}$ Helmholtz Institute Ulm, Helmholtzstraße 11, 89081 Ulm, Germany \\ ${ }^{b}$ Karlsruhe Institute of Technology (KIT), PO Box 3640, 76021 Karlsruhe, Germany \\ ${ }^{c}$ Ulm University, Institute for Theoretical Chemistry, Albert-Einstein-Allee 11, 89081 Ulm, Germany \\ ${ }^{d}$ Department of Mechanical Engineering, Carnegie Mellon University, 5000 Forbes Ave, Pittsburgh, PA 15213, \\ USA
}

\begin{abstract}
The development of new electrolytes is a centerpiece of many strategies to improve electrochemical double layer capacitor (EDLC) devices. We present here a computational screening-based rational design approach to find new electrolyte materials. As an example application, the known chemical space of almost 70 million compounds is investigated in search of electrochemically more stable solvents. Cyano esters are identified as especially promising new compound class. Theoretical predictions are validated with subsequent experimental studies on a selected case. These studies show that based on theoretical predictions only, a previously untested, but very well performing compound class was identified. We thus find that our rational design strategy is indeed able to successfully identify completely new materials with substantially improved properties.
\end{abstract}

Keywords: EDLC, electrolyte materials, solvents, rational design, computational screening

\section{Corresponding authors:}

Martin Korth (martin.korth@uni-ulm.de), Andrea Balducci (andrea.balducci@kit.edu) 


\section{Introduction}

Electrochemical double layer capacitors (EDLCs) are electrochemical energy storage devices with substantial advantages considering cycle life, power density and charging-discharging time . Their most critical property, the operative voltage, is limited by the stability of the electrolyte, which usually consists of quaternary ammonium salts like tetraethylammonium tetrafluoroborate $\left(\mathrm{Et}_{4} \mathrm{NBF}_{4}\right)$ in organic solvents like propylene carbonate $(\mathrm{PC})$ or acetonitrile $(\mathrm{ACN})$. Ionic liquids do allow for higher operative voltages [5], but their high viscosity and low conductivity limits their performance at room temperature (RT). The opportunities offered by organic solvents have furthermore not yet systematically investigated, though very promising results were achieved with for instance adiponitrile (ADN) [9]. One reason is that such studies are very resource intensive when done experimentally, which is why we have recently suggested to integrate computational screening methods with subsequent experimental studies to guide the development of new EDLC electrolyte materials . The application of atom-scale computational methods is not new to the field, but large-scale screening approaches for electrolyte materials have been established only very recently, and only our earlier study presents experimentally validated results for EDLC electrolytes [10].

Our screening approach makes use of methods from Quantum Chemistry, Chemoinformatics and Chemical Engineering to compute not only electrochemical stability window estimates, but also melting/flash/boiling points, viscosities, ion solubilities, pKas and further reactivity measures. Using this approach to screen the 'toy system' of all reasonable, liquid nitrile solvents (almost 5000 compounds), $\mathrm{ACN}$ and $\mathrm{ADN}$ and a number of previously not investigated nitriles were found in the top10 list. Experimental investigation of two of the previously untested nitriles 
in the top10 list showed that also these nitriles performed well as electrolyte solvents [11]. Having a method at hand to pick promising candidates from systematically generated structure databases in a black box fashion, one is now in the position to systematically investigate the opportunities offered for designing new electrolytes based on organic liquids. The most crucial step is the setup of the "chemical space" of possible structures that are fed into the computational screening procedure. This "exploration" of chemical spaces is a well-known problem in computational drug design, where also organic molecules with specific physico-chemical properties are the target, though different properties are important. As a first step to make use of this knowledge for the development of new electrolyte materials, we have recently investigated the complete known chemical space, i.e. all compounds (about 70 million) which were reported to be made by chemists since such information is automatically fed into large databases like the PUBCHEM or CAS databases [22]. Our first analysis focused on identifying stable electrolytes that can provide desired solvation properties of the intermediates involved in lithium-air battery. The computational screening approach outlined above covers all relevant, basic properties of electrolyte solvents. For the case of lithium-air batteries, some of us had already identified a set of fundamental descriptors from this list, such as HOMO level, pKa, free energy of solvation of $\mathrm{Li}^{+}$and $\mathrm{O}_{2}{ }^{-}$, viscosity . These (and many more) descriptors are now easily computable in large scale screenings and have already identified new promising electrolyte candidates. As a next step, we have made the computed data available to the community to explore novel ways for applications in electrochemistry. A key ingredient to enable discovery with the dataset is to provide users with a rich set of interaction and querying abilities. With this in mind, we have developed a tool termed SEED, System for Electrolyte Exploration and Discovery, which 
provides two ways for users to interact with the computed data, (i) visual exploration and (ii) natural language querying. The visual exploration tool allows for similarity and dissimilarity search, i.e. find trends that look similar or dissimilar to ones given by the user, respectively and representative and outlier search, i.e. find trends that are typical or anomalous, respectively. In the natural language querying scheme, users can directly query about the properties in natural language, for e.g. solvents with homo level less than -11.4 and with pKa in DMSO greater than 30. Given an electrolyte must satisfy many criteria simultaneously and often, these criteria are anti-correlated leading to multi-dimensional trade-offs that need to be taken into account when choosing an electrolyte. We believe candidate electrolytes identified through such systematic data-driven discovery will have a much higher likelihood of success than the Edisonian trial-anderror approach pursued currently.

In the following we present an analysis of this database with the goal of identifying new highvoltage electrolyte solvents for EDLCs, as well as experimental results for the validation of our theoretical predictions. This should serve as an example application case to illustrate how our approach can assist the development of new EDLC electrolyte materials and how it can help to gain further insight on organic liquid materials for electrochemistry.

\section{Experimental}

\subsection{Computational Screening}

The following is a short summary of how the data was generated in our previous work, for details see the original publication [22]. Properties that were identified as crucial were: High electrochemical stabilities, suitable melting and boiling points, high flash points, low 
viscosities/high ion conductivities, and high ion solubilities (as well as low toxicity and cost).

Estimates for these properties were computed with quantum chemical methods and the COSMOtherm model, with typical errors of about 5-10\% and excellent correlation ( $\mathrm{R}$ values close or above 0.9) between theoretical predictions and experimental measurements.[10] $\mathrm{pKa}$ values were computed as first measure of the general chemical stability of the compound for the most promising compounds.

Semi-empirical PM6-DH+ calculations were done with MOPAC2012 [31] making use of the COSMO solvation model [32] to generate the input for COSMOtherm (release C30-1501). [32] BP86 [33, 34] and B3LYP [35, 36] DFT calculations have been performed with TURBOMOLE 6.4 [37, 38], using D3 dispersion corrections [39], the RI approximation for two-electronintegrals and again COSMO to generate input for COSMOtherm. Def2-TZVP atomic orbital basis [42] sets were employed throughout for TURBOMOLE calculations. Different conformations and vap. files were not taken into account. Doing so would mean a vast increase of computational work, which - as we mainly operate on semi-empirical level here unreasonable for the purpose of our study.

Structures were obtained from the PubChem Compound database, which comprised 67 million compounds at the time of retrieval. We then applied a hierarchical down-selection strategy to exclude all compounds which cannot be organic liquids due to elemental composition, very large size, etc. After additionally excluding all compounds for which high viscosity estimates were computed, almost 1 million $(927,000)$ polar, organic liquids were left. SQM-level data is available for the full set. DFT-level data for the full set is in preparation and will also be released 
to the public. This set of compounds is analyzed here. Different strategies for doing this are possible, depending on the requirements that prospective new compounds have to meet.

Our final analysis in section 3.1 focuses on suitable solvents with a high electrochemical stability, as we see the operative voltage as the most critical property. The final ranking listed only few commercially available compounds, from which we picked one especially promising candidate (a cyano ester) for experimental validation (see section 3.2 for details) to illustrate the power of rational design approach.

\subsection{Physical and Electrochemical Characterization}

Based on the results of the computational screening, 3-Cyanopropionic acid methyl ester (CPAME, 99\%, ABCR) was chosen for in depth electrochemical investigation. In a first step the solvent was dried over molecular sieve ( $3 \AA$ ) until its water content was below 25 ppm, as measured by Karl-Fischer technique. The conductive salt, tetraethylammonium tetrafluoroborate $\left(\mathrm{Et}_{4} \mathrm{NBF}_{4}\right.$, Sigma-Aldrich), was added to the solvent until the maximum solubility was reached $\left(0.6 \mathrm{M}\right.$ at $\left.20^{\circ} \mathrm{C}\right)$. Both storing of the solvent as well as the electrolyte preparation were done in a glove box (LabMaster, MBRAUN GmbH) with water and oxygen contents below 0.1 ppm.

The conductivity of the electrolyte was determined by performing impedance measurements with a Solartron model 1260 Impedance coupled with a potentiostat/galvanostat 273A PAR. A $0.01 \mathrm{~mol} \mathrm{dm}^{-3}$ aqueous solution of $\mathrm{KCl}$ (VWR) was used to calibrate the sealed glass conductivity cell with platinized Pt electrodes. The temperature dependency of the conductivity for the electrolytes was investigated in the range extending from 20 to $80^{\circ} \mathrm{C}$. 
The viscosity of the electrolytes was measured using an Anton Paar Modular Compact MCR 102 Rheometer, in the same temperature range as the conductivity.

Carbon electrodes were prepared following the procedure reported in Ref. [43] using AC as active material. The ratio of active material (DLC Super 30, Norit), conductive agent (SuperC65, Imerys) and binder (CMC, Walocel CRT 2000 PPA 12 from Dow Wolff Cellulosics) was 90:5:5 with final electrode thickness of $98 \mu \mathrm{m}$ and an electrode diameter of $12 \mathrm{~mm}$. The mass loading of the active material of the electrodes was $5.0 \mathrm{mg} \cdot \mathrm{cm}^{-2}$ average. Free standing electrodes with mass loadings over $30 \mathrm{mg} \cdot \mathrm{cm}^{-2}$ were prepared using a different binder (Polytetrafluoroethylene, PTFE, Sigma Aldrich) with a final composition of 85:10:5 (activated carbon, carbon black, PTFE). The aqueous PTFE solution, the activated carbon and the carbon black were dispersed in an excess amount of ethanol and stirred at elevated temperatures until the slurry became a highly viscous mass. This mass was put onto a glass plate and rolled out several times before punching out disks with a diameter of $12 \mathrm{~mm}$ (area: $1.13 \mathrm{~cm}^{2}$ ). The obtained electrodes were pre-dried in an oven (Binder) set to $80^{\circ} \mathrm{C}$, before drying them for $24 \mathrm{~h}$ under vacuum at $180^{\circ} \mathrm{C}$, in order to remove any residual water.

Electrochemical tests were performed using a VMP multichannel potentiostatic-galvanostatic system (Biologic Science Instruments, France) and a Solartron model 1287A potentiostat, both connected to climatic chambers set to $20^{\circ} \mathrm{C}$ (KBF 115, Binder). The electrochemical investigations were carried out with Swagelok®-type cells, which were assembled in an Argon filled glove box, with water and oxygen contents below $1 \mathrm{ppm}$.

The electrochemical stability window of the electrolyte was evaluated in a three-electrode cell by linear sweep voltammetry (LSV) at $20^{\circ} \mathrm{C}$ using a scan rate of $1 \mathrm{mV} \cdot \mathrm{s}^{-1}$. A platinum 
microelectrode (embedded in PEEK; active area $=0.79 \mathrm{~mm}^{2}$ ) was used as a working electrode and a heavy, free standing AC electrode was used as counter electrode. As a reference electrode, an Ag quasi-reference electrode was employed. To separate the electrodes a Whatman GF/D glass microfiber filter (675 $\mu \mathrm{m}$ thickness and $13 \mathrm{~mm}$ diameter) was used as separator, which was drenched with $150 \mu \mathrm{L}$ of electrolyte. The measurements were performed using a Solartron model 1287A potentiostat controlled by Corrware ${ }^{\circledR}$ software. Separate LSV tests were carried out on each sample to determine the cathodic and anodic electrochemical stability limits. The measurements were performed by scanning the cell potential from the open circuit potential (OCP) towards more negative (cathodic limit) or positive (anodic limit) potentials. Clean electrodes and fresh samples were used for each test.

The maximum operative voltage of the electrolyte was determined using the same threeelectrode cell setup, but instead of a platinum microelectrode an AC composite electrode was utilized as the WE. The measurements were performed with the VMP by cyclic voltammetry (CV), scanning the cell potential with a scan rate of $5 \mathrm{mV} \cdot \mathrm{s}^{-1}$. Starting from a potential of $0.8 \mathrm{~V} /$ $0.8 \mathrm{~V}$ vs. Ag, the maximum potential was increased/decreased stepwise by $0.1 \mathrm{~V}$ until the efficiency of the CVs dropped below $99 \%$.

In order to evaluate the performance of EDLCs with these electrolytes, CV and Galvanostatic charge-discharge cycling were carried out in a 2-electrode configuration using $100 \mu \mathrm{L}$ of electrolyte and two AC composite electrodes with similar masses.

Cyclic voltammetry was carried out using scan rates ranging from $5 \mathrm{mV} \cdot \mathrm{s}^{-1}$ to $200 \mathrm{mV} \cdot \mathrm{s}^{-1}$. The reported values for specific capacitance correspond to the value achieved at the half of the maximum voltage and are referred to the mass of the active material of both electrodes. 
Galvanostatic charge-discharge cycling was carried out using current densities ranging from $0.5 \mathrm{~A} \cdot \mathrm{g}^{-1}$ to $10 \mathrm{~A} \cdot \mathrm{g}^{-1}$. The values of capacitance of the total active material $(\mathrm{C})$, equivalent series resistance (ESR), Coulombic efficiency $(\eta)$, average energy $(E)$ and average power $(P)$ have been calculated as indicated in equations [1]-[5], where I is the applied current, $\mathrm{s}$ is the slope of the discharge curve, $\mathrm{m}$ is the mass of the active material, $\Delta \mathrm{V}$ is the ohmic drop, $\mathrm{A}$ is the area of the electrode, $t_{d}$ and $t_{c}$ are the discharge and charge time, respectively, and the integral refers to the area under the discharge curve between the highest and lowest voltage value.

Float tests were carried out with the aim to assess the stability of the investigated electrolytes. For this test, cells containing the respective electrolytes were charged to $3.2 \mathrm{~V}$ and kept at this voltage for $500 \mathrm{~h}$. In intervals of $20 \mathrm{~h}$ short charge-discharge steps followed by Impedance measurements were performed to determine changes in specific capacitance and ESR. The impedance spectra were recorded with $5 \mathrm{mV}$ ac perturbation in the frequency region from $500 \mathrm{kHz}$ to $10 \mathrm{mHz}$.

$$
\mathrm{C}\left[\mathrm{F} \cdot \mathrm{g}^{-1}\right]=\frac{\mathrm{I}[\mathrm{A}]}{\mathrm{s}\left[\mathrm{V} \cdot \mathrm{s}^{-1}\right] \cdot \mathrm{m}[\mathrm{g}]} \mathrm{C}\left[\mathrm{F} \cdot \mathrm{g}^{-1}\right]=\frac{\mathrm{I}[\mathrm{A}]}{\mathrm{s}\left[\mathrm{V} \cdot \mathrm{s}^{-1}\right] \cdot \mathrm{m}[\mathrm{g}]}
$$

$$
\begin{gathered}
\operatorname{ESR}\left[\Omega \cdot \mathrm{cm}^{2}\right]=\frac{\Delta \mathrm{V}[\mathrm{v}]}{2 \cdot \mathrm{I}[\mathrm{A}]} \cdot \mathrm{A}\left[\mathrm{cm}^{2}\right] \operatorname{ESR}\left[\Omega \cdot \mathrm{cm}^{2}\right]=\frac{\Delta \mathrm{V}[\mathrm{v}]}{2 \cdot \mathrm{I}[\mathrm{A}]} \cdot \mathrm{A}\left[\mathrm{cm}^{2}\right] \\
\eta[\%]=\frac{\mathrm{t}_{\mathrm{d}}[\mathrm{s}]}{\mathrm{t}_{\mathrm{c}}[\mathrm{s}]} \cdot 100 \% \eta[\%]=\frac{\mathrm{t}_{\mathrm{d}}[\mathrm{s}]}{\mathrm{t}_{\mathrm{c}}[\mathrm{s}]} \cdot 100 \%
\end{gathered}
$$

$$
\mathrm{E}_{\text {average }}\left[\mathrm{Wh} \cdot \mathrm{kg}^{-1}\right]=\mathrm{I}[\mathrm{A}] \cdot \int \frac{\mathrm{v}[\mathrm{v}]}{\mathrm{m}[\mathrm{g}] \cdot 3.6} \mathrm{dt}_{\mathrm{d}}[\mathrm{s}] \mathrm{E}_{\text {average }}\left[\mathrm{Wh} \cdot \mathrm{kg}^{-1}\right]=\mathrm{I}[\mathrm{A}] \cdot \int \frac{\mathrm{v}[\mathrm{v}]}{\mathrm{m}[\mathrm{g}] \cdot 3.6} \mathrm{dt}_{\mathrm{d}}[\mathrm{s}]
$$




$$
\mathrm{P}_{\text {average }}\left[\mathrm{kW} \cdot \mathrm{kg}^{-1}\right]=\frac{\mathrm{E}_{\text {average }}[\mathrm{Wh}] \cdot 3.6}{\mathrm{t}_{\mathrm{d}}[\mathrm{s}]} \mathrm{P}_{\text {average }}\left[\mathrm{kW} \cdot \mathrm{kg}^{-1}\right]=\frac{\mathrm{E}_{\text {average }}[\mathrm{Wh}] \cdot 3.6}{\mathrm{t}_{\mathrm{d}}[\mathrm{s}]}
$$

\section{Results and discussion}

\subsection{Computational Screening}

Our data set comprises electrochemical stability windows, melting/flash/boiling points, viscosities and ion solubilities of a range of typical salt ions for about 927,000 possible organic liquids. As mentioned above, this data set is now made available for the community via SEED. From this set we excluded for the following all protic compounds, chloro-compounds, compounds with double bonds, (pure) fluorinated hydrocarbons, peroxides, disulfides, and isothiocyano- and isocyano-compounds. The remaining 'clean' set comprised 289,797 compounds. To illustrate how it can be used to gain insight into the opportunities of molecular materials for electrochemistry, and to assist the development of new EDLC electrolyte materials, we look at both general correlations between different properties and conduct a first systematic search for high-voltage electrolyte solvents.

The most interesting correlations between the different properties are found for viscosities/melting points and for the solubilities of the most important conducting salt ion species. Fig. 1 a shows viscosities and flash points for all $(195,873)$ compounds from the clean set with a viscosity lower than $10 \mathrm{mPa} \cdot \mathrm{s}$. The correlation between viscosities and flash points is high $(\mathrm{R}=0.81)$ and becomes especially problematic for viscosities below $1 \mathrm{mPa} \cdot \mathrm{s}$. If we narrow down our analysis to a single, simple compound classes like nitriles (1650 compounds) or esters (6632 compounds), the correlation is higher ( $\mathrm{R}=0.83$ for both nitriles and esters), see Fig. $1 \mathrm{~b}$ and 
c. But different compound classes show different trends, and the situation thus becomes less assessable to chemical intuition when multi-functional (i.e. containing two or functional groups) compounds are investigated, for instance cyano-esters (1149 compounds, $R=0.75$ ), see Fig. $1 \mathrm{~d}$. This illustrates nicely that chemical intuition and also computational methods should neither be underestimated nor over-valued. A high correlation between viscosity and flash point is well in line what chemical intuition tells us, but the quantification of the trends becomes essentially impossible without computational methods as soon as we include cases of higher complexity.
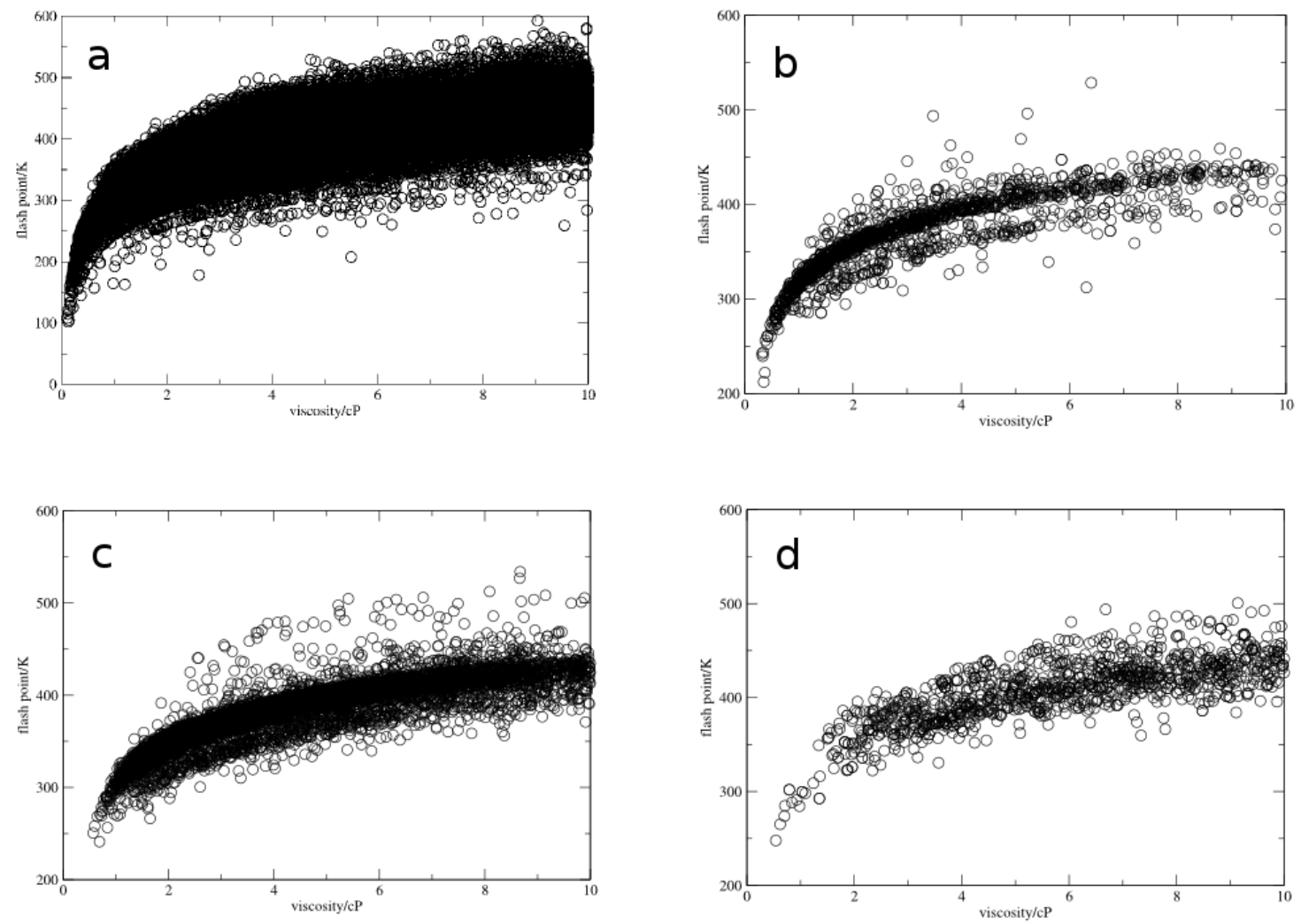

Figure 1. Flash point in $\mathrm{K}$ vs viscosity in $\mathrm{mPa} \cdot \mathrm{s}$ for a) the 'clean' set of almost 300.000 organic liquid compounds, b) all (1650) nitriles of the 'clean' set, c) all (6632) esters of the 'clean' set, and d) all (1149) cyano-esters of the 'clean' set. 
Similar investigations can be made for instance for ion solubilities. Detailed self-studies are possible via the SEED interface, here we summarize only a few interesting findings: Solubilites for all small, spherical cations are highly correlated (with $\mathrm{R}$ values above 0.97 ). Solubilities for large ('softer') anions are also highly correlated, but differ substantially from those of small ('harder') anions. This is well in line with chemical intuition and puts emphasis on the important role of the anionic species for optimizing conducting salts. Though specific (e.g., covalent) salt/solvent interactions are not taken into at the level or the COMSOtherm model with which we compute solubility estimates, we nevertheless believe that the benefit of our database is again the possibility to quantify (and therefore fine-tune) the different relevant properties.

Next we turn to our rational design example. The goal here is to identify suitable high-voltage electrolyte solvents for EDLCs. As the first step we sort out all compounds with an oxidative stability lower (here: HOMO higher) than ethylen carbonate (EC), a viscosity higher than $2.0 \mathrm{mPa} \cdot \mathrm{s}$ or a flash point lower than $333 \mathrm{~K}$. Only 336 compounds fulfill these (very demanding) conditions, so that we can directly proceed to our final analysis, instead of applying more sophisticated filter algorithms. Overall 38 different functional motifs occur in the results set, with many compounds combining several functional motifs. With respect to high oxidative stability, the highest ranked functional motifs are acyl-fluorides $\mathrm{R}-\mathrm{C}(=\mathrm{O}) \mathrm{F}$, carbonates, anhydrides, hetero-cycles, fluorophosphates RO-R(=O)(F)-OR, nitriles, sulfonyl-fluorides, nitro compounds, (organic) nitrates, fluorinated sulfonic acid esters, esters, lactones, and diethers. Subsequent melting point calculations showed that 148 of 336 compounds are liquid at room temperature. A preliminary check (assuming that commercially available compounds obey a regular naming scheme) suggests that 69 of 336 compounds are commercially available. Most likely liquid at 
room temperature and commercially available are only 22 compounds. Among them are PC, ADN, 2-methyl-glutaronitrile (2MGN), glutaronitrile (GTN), 2 fluorophosphates, 2 fluorinated esters, 5 cyano esters (with two being the same substance), 3 additional di-nitriles, 2 linear carbonates, and a lactone. (ACN is not among them due to the restrictive flash point filtering.) Our focus is on non-fluorinated, new (previsouly untested) compound classes, which makes cyano esters the most promising choice. The most promising cyano esters at SQM-level are R1$\mathrm{O}-\mathrm{C}(=\mathrm{O})-\mathrm{R} 2-\mathrm{CN}$ compounds with $\mathrm{Me}$ or $\mathrm{Et}$ as $\mathrm{R} 1$ and $\mathrm{CH}(\mathrm{CH} 3), \mathrm{C}(\mathrm{CH} 3)(\mathrm{CH} 3)$, or $\mathrm{CH}(\mathrm{CH} 3) \mathrm{CH} 2$ as $\mathrm{R} 2$. For final recommendations we nevertheless found it necessary in the past to go to DFT-level estimates and to include pKa estimates to ensure that the general chemical stability is high enough (assuming that this is the case for pKas beyond 24). A DFT-level reevaluation of all cyano esters in the clean set suggested Me as $\mathrm{R} 1$ and $\mathrm{C}(\mathrm{CH} 3(\mathrm{CH} 3)$ or $\mathrm{CH} 2 \mathrm{CH} 2$ as R2 as optimal choice. The second compound, 3-Cyanopropionic acid methyl ester (CPAME), was chosen for subsequent experimental tests.

The above outlined analysis does not only illustrate the advantages of our rational design approach, it also shows the limitations of the known chemical space. This is depicted in Fig. 2. The current standard electrolyte solvents are close to the best what the known chemical space offers. Important new impulses should be expected first of all from a) exploring the unknown chemical space, especially concerning multifunctional compounds, b) the systematic exploration of mixtures, and c) optimizing salt/solvent combinations. Our rational design approach is ideally suited to address all three options in a systematic fashion, as it allows for a quantitative assessment of the relevant properties. Though the search example we show in the article is 


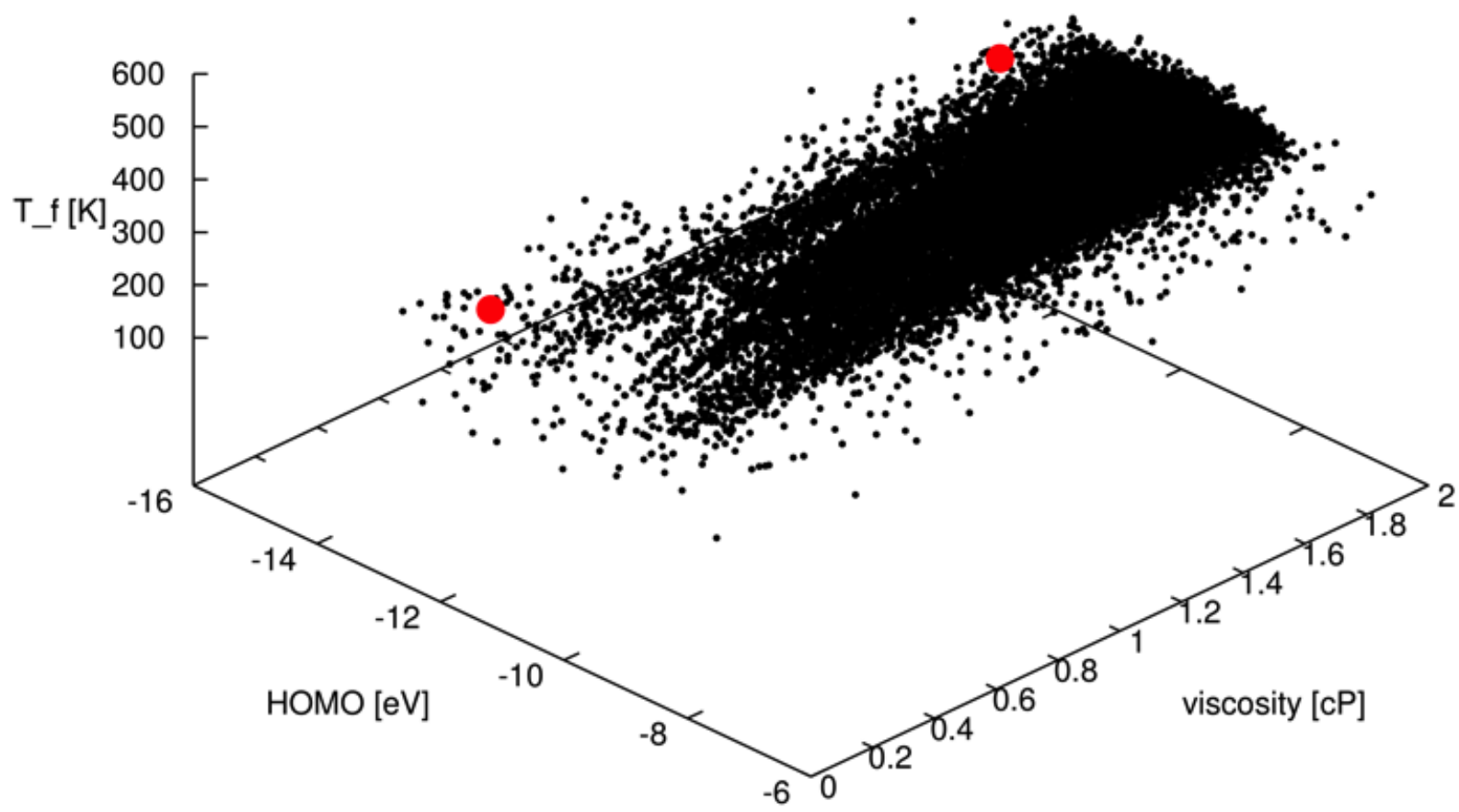

Figure 2. Flash point in $\mathrm{K}$ vs viscosity in $\mathrm{mPa} \cdot \mathrm{s} v \mathrm{HOMO}$ in $\mathrm{eV}$ for all compounds from the 'clean' set with a viscosity below $2 \mathrm{mPa} \cdot \mathrm{s}$. Red dots mark ACN and PC.

\subsection{Physical \& Electrochemical Characterization}

Based on the results of the computational screening, 3-Cyanopropionic acid methyl ester (CPAME) was chosen for an in depth electrochemical characterization. Table 1 reports the chemical structure, purity and maximum concentration of $\mathrm{Et}_{4} \mathrm{NBF}_{4}$ in this solvent. The maximum concentration was found to be $0.6 \mathrm{M}$. 


\begin{tabular}{|c|c|c|c|c|c|c|c|}
\hline Name & Purity / \% & $\begin{array}{c}\mathbf{c}_{\mathrm{Et} 4 \mathrm{NBF} 4} / \\
\mathrm{mol} \mathrm{dm}^{-3}\end{array}$ & $\sigma / \mathrm{mS} \cdot \mathrm{cm}^{-1}$ & $\begin{array}{c}\mathbf{T}_{\mathbf{0}, \boldsymbol{\sigma}}{ }^{\prime} \\
\mathbf{K}\end{array}$ & $\begin{array}{c}\sigma_{0} / \mathrm{ms}^{\cdot \mathrm{cm}^{-}} \\
1\end{array}$ & $\begin{array}{c}\mathbf{B}_{\boldsymbol{\sigma}}{ }^{\prime} \\
\mathbf{K}\end{array}$ & $\mathbf{R}^{2}$ \\
\hline $\begin{array}{l}\text { 3-cyano- } \\
\text { propionic acid } \\
\text { methyl ester }\end{array}$ & 99 & 0.6 & 4.3 & 178 & 104.6 & 367.3 & 0.99994 \\
\hline & Structure & & $\eta / \mathrm{mS} \cdot \mathrm{cm}^{-1}$ & $\begin{array}{c}\mathbf{T}_{\mathbf{0 , \boldsymbol { \eta }}}{ }^{\prime} \\
\mathbf{K}\end{array}$ & $\boldsymbol{\eta}_{\mathbf{0}} / \mathbf{m P a} \cdot \mathbf{s}$ & $\begin{array}{c}\mathbf{B}_{\boldsymbol{\eta}}{ }^{\prime} \\
\mathbf{K}\end{array}$ & $\mathbf{R}^{2}$ \\
\hline & & & 5.5 & 190 & 0.24 & 332.3 & 0.99994 \\
\hline
\end{tabular}

Table 1. Purity, concentration of $\mathrm{Et}_{4} \mathrm{NBF}_{4}$, structure, conductivity and viscosity at $20^{\circ} \mathrm{C}$ as well as VTF fitting parameters of the investigated cyano ester.

Among other features, good transport properties, i.e. high conductivity and low viscosity, are considered of great importance for novel electrolytes. The conductivity and viscosity of the considered electrolyte were investigated in the temperature range between 20 and $80^{\circ} \mathrm{C}$ (Fig. 3). Lower temperatures were not considered, because for temperature below $20^{\circ} \mathrm{C}$ the conducting salt starts to crystalize. As seen in Fig. $3 b$ and $d$, the temperature dependency of both conductivity and viscosity could be very well described by the Vogel-Tammann-Fulcher (VTF) equation. The corresponding VTF parameters as well as the conductivity and viscosity values at $20^{\circ} \mathrm{C}$ are reported in Table 1 . At $20^{\circ} \mathrm{C}$ the electrolyte has a conductivity $4.3 \mathrm{mS} \mathrm{cm}^{-1}$ of and a viscosity of $5.5 \mathrm{mPa} \cdot \mathrm{s}$. In our previous publication we considered different nitrile solvents (glutaronitrile, 2-methylglutaronitrile and adiponitrile) as electrolytes after computational screening [11]. Using the same conductive salt $\left(\mathrm{Et}_{4} \mathrm{NBF}_{4}\right)$, as in this study, conductivity and 
viscosity values in the range of $2.3-6.1 \mathrm{mS} \cdot \mathrm{cm}^{-1}$ and $8.0-9.5 \mathrm{mPa} \cdot \mathrm{s}$, respectively, were achieved. Compared to these values, the cyano ester considered in this study reaches a similar conductivity, while displaying lower viscosity at the same time. Compared to the state-of-the-art electrolytes like $1 \mathrm{MEt}_{4} \mathrm{NBF}_{4}$ in $\mathrm{ACN}$ or $1 \mathrm{MEt}_{4} \mathrm{NBF}_{4}$ in $\mathrm{PC}$, which have a conductivity and viscosity of $55 \mathrm{mS} \cdot \mathrm{cm}^{-1} / 0.6 \mathrm{mPa} \cdot \mathrm{s}$ and $13 \mathrm{mS} \cdot \mathrm{cm}^{-1} / 2.6 \mathrm{mPa} \cdot \mathrm{s}$, respectively, the considered electrolyte has a lower conductivity and higher viscosity, especially when compared to ACN. Most likely, the low conductivity of the cyano ester is related to the low concentration of the conducting salt of $0.6 \mathrm{M}$.
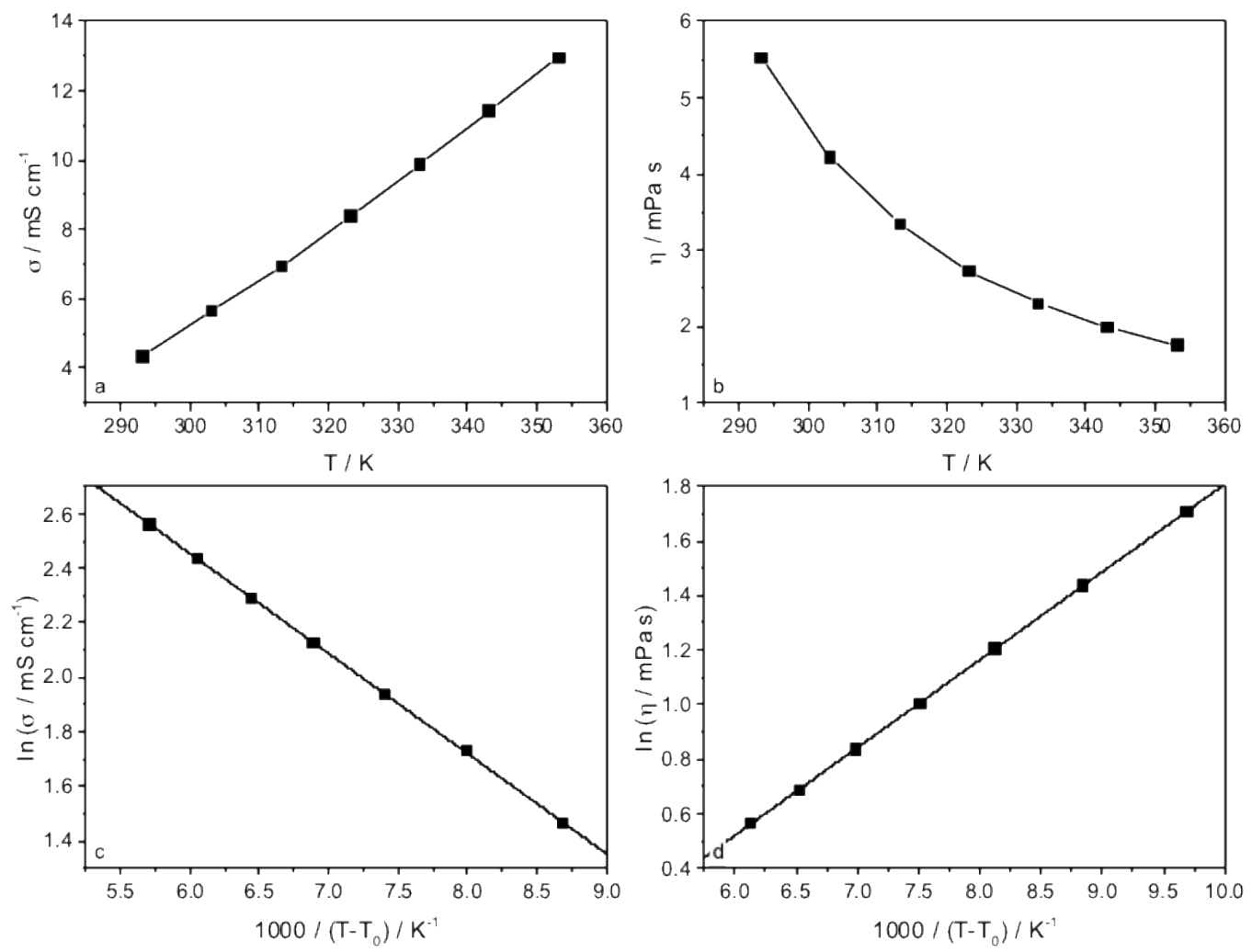

Figure 3. (a) Conductivity and (b) viscosity of the $0.6 \mathrm{M} \mathrm{Et}_{4} \mathrm{NBF}_{4}$ in 3-cyano-propionic acid methyl ester in the temperature range of $20-80^{\circ} \mathrm{C}$ with the corresponding VTF plots $(\mathrm{c}, \mathrm{d})$. 
The electrochemical stability window (ESW) of the electrolyte can be seen in Fig. 4. The cathodic stability of the electrolyte is $-1.5 \mathrm{~V}$ vs. Ag. On the anodic side a peak at $3.2 \mathrm{~V}$ vs. Ag can be observed which could be related to impurities of the solvent, since it was used as received without any purification before use. For higher potentials than $3.2 \mathrm{~V}$ the measured current decreases slightly and at a potential of $3.9 \mathrm{~V}$ vs. Ag the current increases sharply, indicating the start of the full decomposition of the electrolyte.

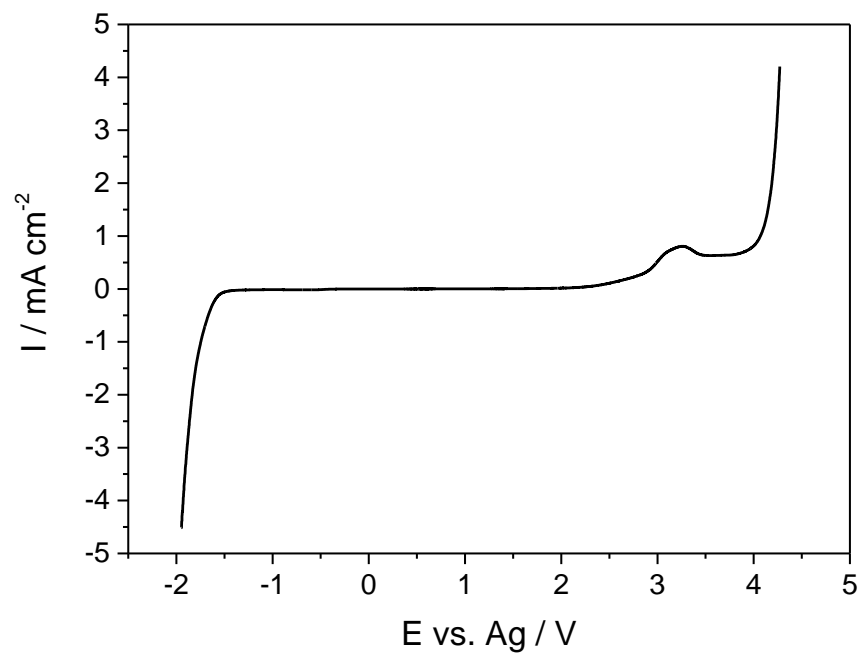

Figure 4. Electrochemical stability windows of the considered $0.6 \mathrm{M} \mathrm{Et}_{4} \mathrm{NBF}_{4}$ in 3-cyanopropionic acid methyl ester.

After the determination of the ESW, the operative voltages of the electrolytes were determined via conducting 3-electrode setup CV measurements. Fig. 5 presents the obtained CV curves as well as the obtained efficiencies. The positive and negative potential limits were defined by the highest and lowest potential limits, at which the efficiency of the charge-discharge was higher 
than $99 \%$. Using these thresholds, the electrolyte is stable with the used activated carbon up to 2.0 V vs. Ag on the positive side and up to $-1.8 \mathrm{~V}$ on the negative side. Thus, the maximum operative voltage can be calculated to $3.8 \mathrm{~V}$, which is much higher than the maximum voltage usually reported for organic solvent based electrolytes. However, a usable voltage of $3.7 \mathrm{~V}$ has already been reported for other nitrile based organic solvents like adiponitrile [9].

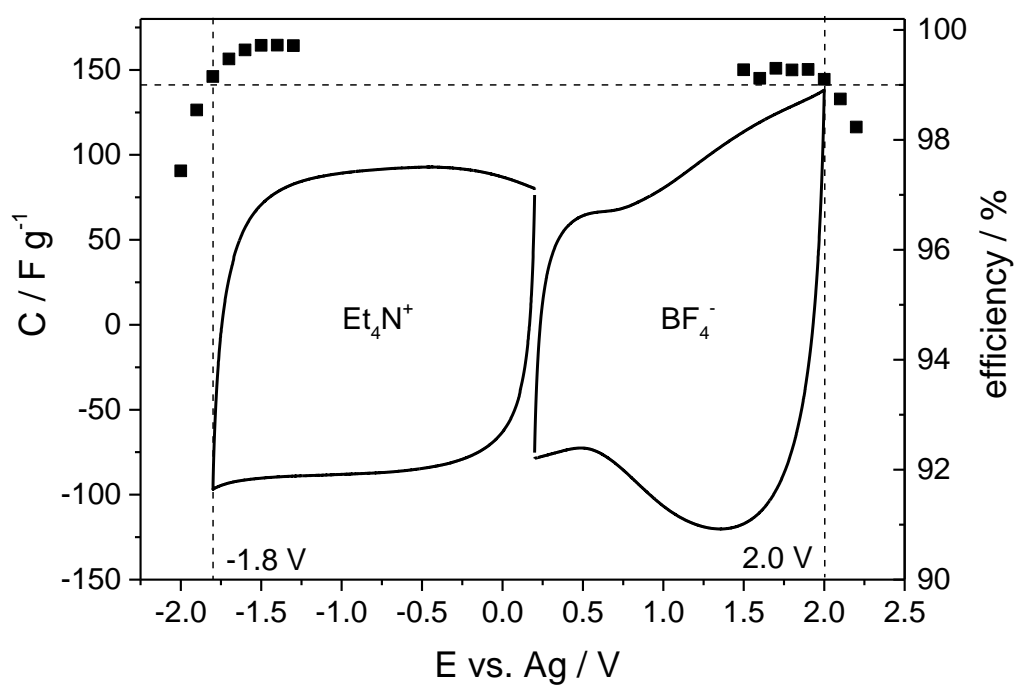

Figure 5. Specific capacitance (line) and Coulombic efficiency (scatter) obtained by CV at $5 \mathrm{mV} \mathrm{s}^{-1}$ using an $\mathrm{AC}$ electrode and $0.6 \mathrm{M} \mathrm{Et}_{4} \mathrm{NBF}_{4}$ in 3-cyano-propionic acid methyl ester as electrolyte. The horizontal line represents a $99 \%$ threshold in efficiency, the vertical lines mark the potential at which the efficiency falls below $99 \%$.

In order to assess the cycling performance of the electrolyte, full cells were assembled using a symmetric configuration. Using this configuration, not the full potential window can be used due to the asymmetrical voltage excursion on the positive and negative side. Therefore, in order to 
avoid premature electrolyte decomposition, the cells were only cycled up to a maximum potential of $3.5 \mathrm{~V}$.

Fig. 6a shows exemplary a CV for the investigated electrolyte for a scan rate of $20 \mathrm{mV} \cdot \mathrm{s}^{-1}$. As visible, the $\mathrm{CV}$ has the typical rectangular shape for capacitive behavior and no peaks can be observed, which would indicate unwanted faradaic reactions. Using this scan rate, a specific capacitance of $25 \mathrm{~F} \cdot \mathrm{g}^{-1}$ is reached for the whole device. Fig. $6 \mathrm{~b}$ shows the dependency of the specific capacitance on the applied scan rate. At a scan rate of $200 \mathrm{mV} \cdot \mathrm{s}^{-1} 46 \%$ of the initial capacitance at $5 \mathrm{mV} \cdot \mathrm{s}^{-1}$ is left. As mentioned above, the conductivity of the electrolyte is only $4.3 \mathrm{mS} \cdot \mathrm{cm}^{-1}$ which leads to a higher equivalent series resistance (compared to other organic solvent-based electrolytes, like ACN or PC), and therefore to a lower high rate performance.
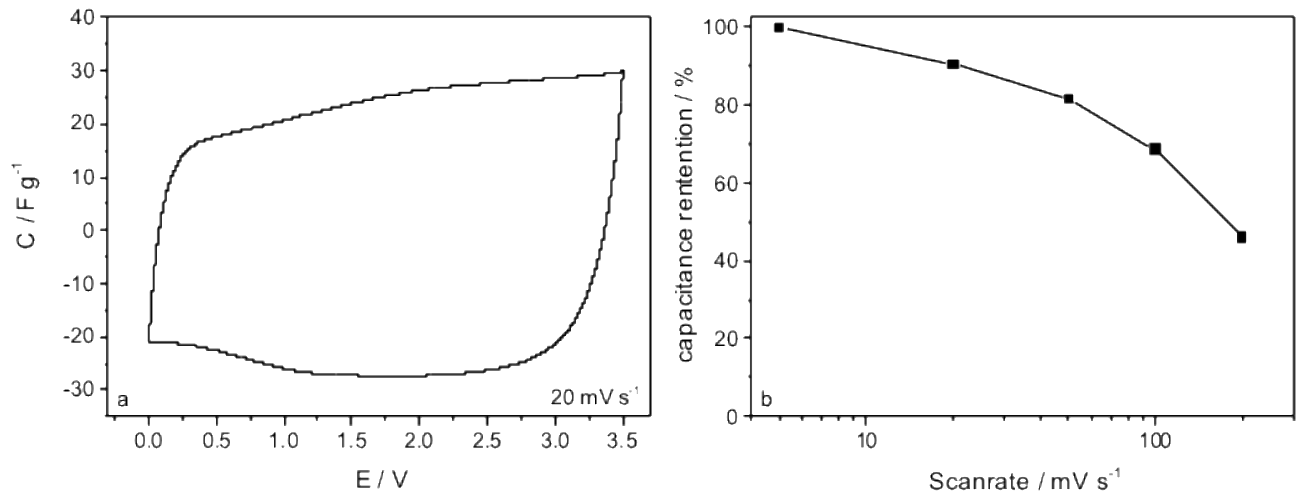

Figure 6. (a) Cyclic voltammetry of the investigated electrolyte at a scan rate of $20 \mathrm{mV} \mathrm{s}^{-1}$. (b) Capacitance retention for scan rates ranging from 5 to $200 \mathrm{mV} \mathrm{s}^{-1}$.

To further investigate the performance of the electrolytes, galvanostatic cycling experiments were performed using current densities ranging from $0.5 \mathrm{~A} \cdot \mathrm{g}^{-1}$ to $5 \mathrm{~A} \cdot \mathrm{g}^{-1}$ with a maximum 
applied voltage of $3.5 \mathrm{~V}$ (Fig. 7). Both the Coulombic efficiency as well as the ESR are stable upon cycling indicating that no severe electrolyte degradation is taking place. The efficiency reaches values close to $100 \%$ and the ESR reaches values around $16.5 \Omega \cdot \mathrm{cm}^{2}$. During the first 300 cycles the efficiency increases slightly and at the same time the capacitance decreases from 25.7 $\mathrm{F} \cdot \mathrm{g}^{-1}$ to $22.3 \mathrm{~F} \cdot \mathrm{g}^{-1}$ at a current of $0.5 \mathrm{~A} \cdot \mathrm{g}^{-1}$. For higher currents the specific capacitance stabilizes. A possible explanation of the initial loss could be that the cell is operating slightly outside of the electrolytes' stability window. At higher currents, however, the time the cell spends outside of the stability window of the electrolyte is not sufficient enough to allow the electrolyte degradation.

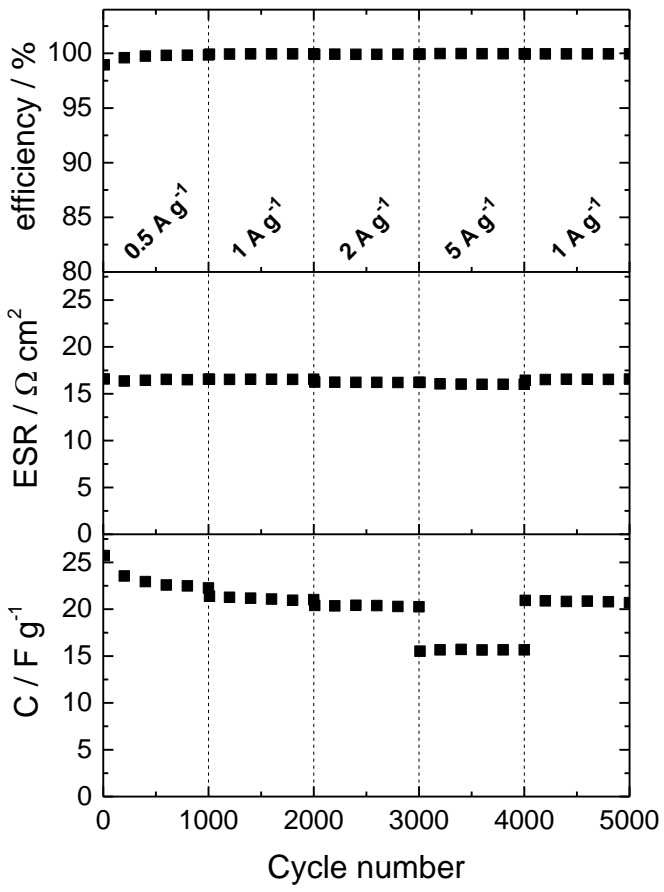

Figure 7. Evolution of specific capacitance, ESR and Coulombic efficiency of EDLCs containing the investigated electrolyte using a maximum of $3.5 \mathrm{~V}$ and current densities from 0.5 to $10 \mathrm{~A} \cdot \mathrm{g}^{-1}$. 
Finally, float tests were carried out as a stress test, keeping the cells charged at $3.2 \mathrm{~V}$ for $500 \mathrm{~h}$ (Fig. 8). In intervals of $20 \mathrm{~h}$, short charge/discharge steps were performed to follow the development of capacitance and ESR. Starting with a specific capacitance of $20.3 \mathrm{~F} \cdot \mathrm{g}^{-1}$ and an ESR of $16.5 \Omega \cdot \mathrm{cm}^{2}$, after $500 \mathrm{~h}$ of float test still $78.3 \%$ of the initial capacitance are left $\left(15.9 \mathrm{~F} \cdot \mathrm{g}^{-1}\right)$. In the same time frame the ESR increases slightly to $18.6 \Omega \cdot \mathrm{cm}^{2}(112.7 \%)$. These results clearly demonstrate the stability of the electrolyte system and indicate CPAME as a very interesting solvent for the realization of high voltage EDLCs.

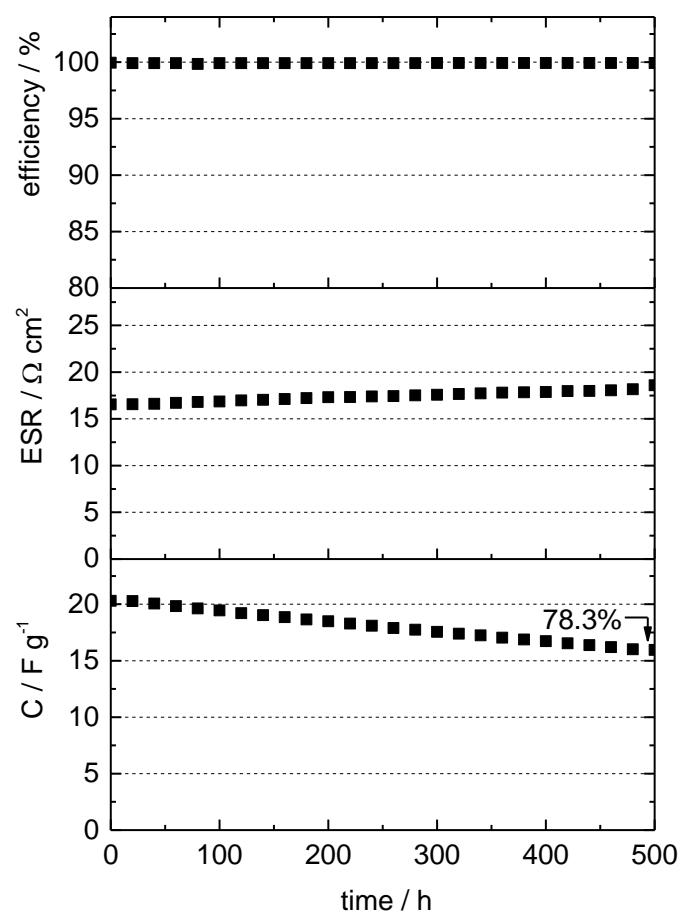

Figure 8. Evolution of specific capacitance, ESR and efficiency for $500 \mathrm{~h}$ float test. 


\section{Conclusion}

The introduction of advanced electrolytes is one of the keys for the development of high energy EDLCs. To assist the development of new EDLC electrolyte materials, we applied a computational screening-based rational design approach to identify new high-voltage solvents. This approach was based on searching the complete known chemical space for new compound classes with advantageous combinations of electrochemical stability windows melting/flash/boiling points, viscosities, ion solubilities, and pKas, which were computed at either SQM or DFT level and subsequent COSMOtherm calculations. Our analysis suggested a number of compound classes well in line with the known literature (carbonates, (di-)nitriles, dinitriles), but also some compound classes and combinations thereof that were not yet suggested as EDLC solvents. Among these latter classes, cyano esters, especially the 3-Cyanopropionic acid methyl ester (CPAME), appear as one of the most promising because of their well-balanced set of chemical-physical properties.

We showed that the use of electrolyte containing CPAME as solvent and $\mathrm{Et}_{4} \mathrm{NBF}_{4}$ as conductive salt allows for the realization of EDLCs with operative voltage as high as $3.5 \mathrm{~V}$. These high voltage EDLCs display good performance in term of specific capacitance and capacitance retention. Furthermore, we showed that CPAME-based EDLCs are able to retain almost $80 \%$ of their initial capacitance after $500 \mathrm{~h}$ of float tests carried out at $3.2 \mathrm{~V}$. This extraordinary capacitance retention, which is among the highest so far reported for high voltage EDLC, clearly indicate that CPAME can be considered as a new and very interesting solvent in view of the realization of high voltage EDLC. 
Taking these results into account, the use of computational screening appears as a very promising and novel strategy for a rational design of new electrolyte materials for EDLCs. It is important to remark that such approach can be applied not only to the search for new materials, but also to the optimization of mixtures and salt/solvent combinations.

Acknowledgements

$\mathrm{AB}$ and $\mathrm{CS}$ would like to thank the Bundesministerium für Bildung and Forschung (BMBF) within the project IES (contract number 03EK3010) for the financial support. We gratefully appreciated the supply of materials by Norit Activated Carbon Holding (DLC Super 30) and Imerys (Super C65). MK and TH gratefully acknowledge financial support from the Barbara Mez-Starck Foundation and the DFG Research Unit FOR1376. This research was supported in part by the bwHPC initiative and the bwHPC-C5 project through compute services of the JUSTUS HPC facility at the University of Ulm. bwHPC and bwHPC-C5 (http://www.bwhpcc5.de) are funded by the Ministry of Science, Research and the Arts Baden-Wurttemberg (MWK) and the Germany Research Foundation (DFG). VV gratefully acknowledges support from the National Science Foundation CAREER award CBET-1554273. 


\section{References}

[1] A.G. Pandolfo, A.F. Hollenkamp, Journal of Power Sources, 157 (2006) 11-27.

[2] P. Simon, Y. Gogotsi, Nat Mater, 7 (2008) 845-854.

[3] P.W. Ruch, D. Cericola, A. Foelske, R. Kotz, A. Wokaun, Electrochim Acta, 55 (2010) 2352-2357.

[4] P.W. Ruch, D. Cericola, A. Foelske-Schmitz, R. Kotz, A. Wokaun, Electrochim Acta, 55 (2010) 4412-4420.

[5] F. Béguin, E. Frąckowiak, Supercapacitors Materials, Systems, and Applications, Wiley-VCH, Weinheim, 2013.

[6] P. Azais, L. Duclaux, P. Florian, D. Massiot, M.A. Lillo-Rodenas, A. Linares-Solano, J.P. Peres, C. Jehoulet, F. Beguin, Journal of Power Sources, 171 (2007) 1046-1053.

[7] M. Conte, Fuel Cells, 10 (2010) 806-818.

[8] J.R. Miller, A.F. Burke, Electrochem. Soc. Interface, 17 (2008) 53-57.

[9] A. Brandt, P. Isken, A. Lex-Balducci, A. Balducci, Journal of Power Sources, 204 (2012) 213-219.

[10] T. Husch, N.D. Yilmazer, A. Balducci, M. Korth, Phys Chem Chem Phys, 17 (2015) 3394-3401.

[11] C. Schütter, T. Husch, M. Korth, A. Balducci, The Journal of Physical Chemistry C, 119 (2015) 13413-13424.

[12] P.B. Balbuena, Y. Wang, Lithium-ion Batteries: Solid-electrolyte Interphase, Imperial College Press, London, 2004.

[13] G. Ferguson, L.A. Curtiss, Atomic-Level Modeling of Organic Electrolytes in Lithium-Ion Batteries, in: Applications of Molecular Modeling to Challenges in Clean Energy, American Chemical Society, 2013, pp. 217233.

[14] K. Leung, The Journal of Physical Chemistry C, 117 (2013) 1539-1547.

[15] K. Leung, Chem Phys Lett, 568-569 (2013) 1-8.

[16] M.D. Bhatt, C. O'Dwyer, Phys Chem Chem Phys, 17 (2015) 4799-4844.

[17] M. Korth, Computational studies of solid electrolyte interphase formation, in: Chemical Modelling: Volume 11, The Royal Society of Chemistry, 2015, pp. 57-87.

[18] M. Korth, Phys Chem Chem Phys, 16 (2014) 7919-7926.

[19] M. Korth, S. Grimme, M.D. Towler, J Phys Chem A, 115 (2011) 11734-11739.

[20] L. Cheng, R.S. Assary, X. Qu, A. Jain, S.P. Ong, N.N. Rajput, K. Persson, L.A. Curtiss, The Journal of Physical Chemistry Letters, 6 (2015) 283-291.

[21] O. Borodin, M. Olguin, C.E. Spear, K.W. Leiter, J. Knap, Nanotechnology, 26 (2015) 354003.

[22] T. Husch, M. Korth, Phys Chem Chem Phys, 17 (2015) 22596-22603.

[23] T. Husch, M. Korth, Phys Chem Chem Phys, 17 (2015) 22799-22808.

[24] A. Khetan, H. Pitsch, V. Viswanathan, J Phys Chem Lett, 5 (2014) 1318-1323.

[25] A. Khetan, H. Pitsch, V. Viswanathan, J Phys Chem Lett, 5 (2014) 2419-2424.

[26] A. Khetan, A. Luntz, V. Viswanathan, J Phys Chem Lett, 6 (2015) 1254-1259.

[27] C.M. Burke, V. Pande, A. Khetan, V. Viswanathan, B.D. McCloskey, P Natl Acad Sci USA, 112 (2015) 92939298.

[28] N.B. Aetukuri, B.D. McCloskey, J.M. Garcia, L.E. Krupp, V. Viswanathan, A.C. Luntz, Nat Chem, 7 (2015) $50-56$.

[29] M. Korth, M. Pitoňák, J. Řezáć, P. Hobza, Journal of Chemical Theory and Computation, 6 (2009) 344-352.

[30] M. Korth, Journal of Chemical Theory and Computation, 6 (2010) 3808-3816.

[31] OPENMOPAC, www.openmopac.net (accessed 15.07.2014).

[32] A. Klamt, Wiley Interdisciplinary Reviews: Computational Molecular Science, 1 (2011) 699-709.

[33] A.D. Becke, Phys Rev A, 38 (1988) 3098-3100.

[34] J. Perdew, Phys Rev B, 33 (1986) 8822-8824.

[35] A.D. Becke, The Journal of Chemical Physics, 98 (1993) 5648.

[36] P.J. Stephens, F.J. Devlin, C.F. Chabalowski, M.J. Frisch, The Journal of Physical Chemistry, 98 (1994) 1162311627.

[37] R. Ahlrichs, M. Bär, M. Häser, H. Horn, C. Kölmel, Chem Phys Lett, 162 (1989) 165-169.

[38] TURBOMOLE, V6.4 2012, http://www.turbomole.com, a development of University of Karlsruhe and Forschungszentrum Karlsruhe GmbH,1989-2007,TURBOMOLE GmbH, since 2007.

[39] S. Grimme, Journal of Computational Chemistry, 27 (2006) 1787-1799.

[40] K. Eichkorn, O. Treutler, H. Öhm, M. Häser, R. Ahlrichs, Chem Phys Lett, 242 (1995) 652-660. 
[41] K. Eichkorn, F. Weigend, O. Treutler, R. Ahlrichs, Theor Chem Acta, 97 (1997) 119-124.

[42] A. Schäfer, C. Huber, R. Ahlrichs, J Chem Phys, 100 (1994) 5829.

[43] A. Krause, P. Kossyrev, M. Oljaca, S. Passerini, M. Winter, A. Balducci, Journal of Power Sources, 196 (2011) 8836-8842.

[44] S. Pohlmann, A. Balducci, Electrochim Acta, 110 (2013) 221-227. 